\title{
Development and Validation of a Stability Indicating RP-HPLC Method for Simultaneous Estimation of Aliskiren Hemifumarate, Amlodipine Besylate and Hydrochlorothiazide in Bulk and Pharmaceutical Dosage Forms
}

\author{
Prathyusha W, Anandkumar R. Tengli*, Chandan R S, Gurupadayya B M, \\ Sai Thanuja V \\ Department of Pharmaceutical Chemistry, JSS College of Pharmacy, JSS University, Sri Shivarathreeshwara \\ Nagar, Mysore-570015, India
}

\begin{abstract}
A simple, selective and precise RP-HPLC method was developed for the simultaneous estimation of Aliskiren Hemifumarate, amlodipine besylate and hydrochlorothiazide in the Bulk and Pharmaceutical Dosage Forms using losartan as an internal standard. The chromatographic separation of the three drugs was achieved on a reverse phase Inertsil-ODS, C18, 100X $4.6 \mathrm{~mm}, 5 \mu \mathrm{m}$ column using $0.1 \mathrm{M}$ Ammonium acetate buffer ( $\mathrm{pH}$ adjusted to 5 using formic acid) and Acetonitrile in the ratio of 65:35 v/v with flow rate of $1.0 \mathrm{ml} / \mathrm{min}$ with injection volume $20 \mu \mathrm{L}$ and the detection was carried out at $232 \mathrm{~nm}$. The retention time of aliskiren hemifumarate (ALSK), amlodipine besylate (AMLO) and hydrochlorothiazide (HCT) were found to be 3.90, 5.22 and $1.91 \mathrm{~min}$ respectively. The drug products were subjected to stress conditions of acidic, alkaline, oxidation, UV and Thermal conditions. The degradation products were well resolved from ALSK, AMLO and HCT peaks, thus indicating the stability-indicating nature of the method. The linear regression analysis data for the calibration plots showed good linear relationship in the concentration range of 37.5-225.00 $\mu \mathrm{g} / \mathrm{ml}$ for aliskiren hemifumarate, 3.125-18.75 $\mu \mathrm{g} / \mathrm{ml}$ for hydrochlorothiazide and 1.25-7.50 $\mu \mathrm{g} / \mathrm{ml}$ for amlodipine besylate. The developed method was successfully validated in accordance to ICH guidelines. Hence, this method can be conveniently adopted for the routine analysis in quality control laboratories.
\end{abstract}

Key words: Aliskiren hemifumarate, Amlodipine besylate, Hydrochlorothiazide, RP-HPLC

\section{Introduction}

Aliskiren, (2(S), 4(S), 5(S), 7(S)-N- (2-carbamoyl- 2- methylpropyl) -5-amino-4-hydroxy2,7 diisopropyl-8-[4-methoxy-3-(3-methoxypropoxy)phenyl] octanamide hemifumarate) 1-3 (Fig. 1). The first oral direct renin inhibitor approved for clinical use, exhibits a novel and advantageous pharmacokinetic and pharmacodynamic profile for the long-term treatment of hypertension. Aliskiren blocks the renin system at its rate-limiting step by directly inhibiting the catalytic activity of renin, thereby reducing generation of angiotensin I and angiotensin II.

Amlodipine, 2[(2-aminoethoxy) methyl]-4-(2-chloro-phenyl)-1, 4-dihydro-6-methyl-3, 5-pyridine carboxylic acid, 3-ethyl, 5-methylester is a dihydro pyridine (Fig. 2) derivative with calcium antagonist activity. It is used in the management of hypertension, chronic stable angina pectoris and prinzmetal variant angina. Amlodipine inhibits the trans-membrane influx of calcium ions into vascular smooth muscle and cardiac muscle.

Hydrochlorothiazide belongs to Thiazide class of diuretics, acting on the kidneys to reduce sodium (Na) reabsorption in the distal convoluted tubule. This increases the osmolarity in the lumen, causing less water to be reabsorbed by the collecting ducts. This leads to increase urinary output. It is chemically 6-chloro-1, 1dichloro-3, 4,dihydro -2H-1, 2, 4-benzoliadiazine-7-sulphanomide1, 1-dioxide (Fig. 3).

Literature survey revealed $\mathrm{HPLC}^{[1]}$, LC-MS ${ }^{[2]}$, spectroflurimetric and simultaneous UVspectrophotometric methods are reported for the estimation of aliskiren hemifumarate ${ }^{[3-4]}$, amlodipine ${ }^{[5-10]}$, hydrochlorothiazide ${ }^{[11-15]}$ alone or in combination with other anti-hypertensive agents. So the present study aim to develop a simple, selective and precise RP-HPLC method was developed for the simultaneous estimation of Aliskiren hemifumarate, amlodipine besylate and hydrochlorothiazide in bulk drug and in combined dosage form. 
<smiles>COCCCOc1cc(CC(CNCC(O)CC(C(=O)NCC(C)(C)C(N)=O)C(C)C)C(C)C)ccc1OC</smiles>

Fig. 1 Structure of Aliskiren<smiles>CCOC(=O)C1=C(COCCN)NC(C)=C(C(=O)OC)C1c1ccccc1Cl</smiles>

Fig. 2 Structure of Amlodipine<smiles>NS(=O)(=O)c1cc2c(cc1Cl)NCNS2(=O)=O</smiles>

Fig. 3 Structure of Hydrochlorothiazide

\subsection{Chromatographic Conditions}

\section{Materials And Methods}

The Waters HPLC with PDA detector and Empower 2 software was employed for the present study. The chromatography determination performed at ambient temperature by using Inertsil-ODS, C18, $100 \mathrm{X} 4.6$, $5 \mu \mathrm{m})$ column, with a mobile phase composed of $0.1 \mathrm{M}$ ammonium acetate: Acetonitrile in the ratio $65: 35(\mathrm{v} / \mathrm{v})$. $\mathrm{pH}$ of the buffer was adjusted to 5 using formic acid. The chromatography run time was maintained up to 10.0 min with flow rate at $1.0 \mathrm{~mL} /$ min with injection volume $20 \mu \mathrm{L}$ and the eluent was monitored at $232 \mathrm{~nm}$.

\subsection{Reference Standards, Reagents}

The standard Aliskiren hemifumarate was obtained from Mylan Pharmaceuticals, Hyderabad. Amlodipine and Hydrochlorothiazide were procured from Life Care Pharmaceutical Industries, Pondicherry. Amturnide tablets were procured from US market. Acetonitrile and water employed for the preparation of mobile phase were of HPLC grade was obtained from Merck limited, Mumbai.

\subsection{Preparation of standard solutions}

Weigh and transfer $12.5 \mathrm{mg}$ of Hydrochlorothiazide, $150 \mathrm{mg}$ of Aliskiren and $5 \mathrm{mg}$ of Amlodipine working standard into $100 \mathrm{~mL}$ volumetric flask, add $50 \mathrm{~mL}$ of diluent and sonicate and make up to the volume with diluent. Transfer $10 \mathrm{~mL}$ of standard stock solution into $100 \mathrm{~mL}$ volumetric flask and dilute it with diluent.

\subsection{Preparation of Sample Solutions}

20 tablets (Amturnide) were taken and finely powdered. Powder equivalent to $12.5 \mathrm{mg}$ of Hydrochlorothiazide, $150 \mathrm{mg}$ of Aliskiren and $5 \mathrm{mg}$ of Amlodipine was taken in to a $100 \mathrm{~mL}$ volumetric flask add $50 \mathrm{~mL}$ of diluent, sonicate for 10 minutes and make up the volume. Further filter the solution through $0.45 \mu$ membrane filter paper. Dilute $10 \mathrm{ml}$ of filtrate to $100 \mathrm{ml}$ with mobile phase.

\subsection{Assay procedure}

In case of marketed formulations, twenty tablet were taken and finely powdered. Powder equivalent to $12.5 \mathrm{mg}$ of Hydrochlorothiazide, $150 \mathrm{mg}$ of Aliskiren and $5 \mathrm{mg}$ of Amlodipine were taken in to a $100 \mathrm{~mL}$ volumetric flask add $50 \mathrm{~mL}$ of diluent, sonicate for 10 minutes and make up the volume.The column was equilibrated for $30 \mathrm{~min}$, with the mobile phase flowing through the system with a flow rate of $1.0 \mathrm{ml} / \mathrm{min}$ and detector was set at a wavelength of $232 \mathrm{~nm}$. The retention time of aliskiren hemifumarate, amlodipine besylate and hydrochlorothiazide were found to be $3.90,5.22,1.91 \mathrm{~min}$ (Fig. 4) respectively in bulk drug and the 
retention time of aliskiren hemifumarate, amlodipine besylate and hydrochlorothiazide were found to be 3.90 , 5.22, 1.91 min (Fig. 5) respectively in pharmaceutical formulation. The blank chromatogram is shown in Fig. 6. The $\%$ purity of aliskiren hemifumarate, amlodipine besylate and hydrochlorothiazide in tablet dosage form were compiled and reported in "Table 1".

Table 1: Determination of ALSK, AMLO and HCT in Tablet dosage form

\begin{tabular}{|l|c|c|c|}
\hline \multicolumn{1}{|c|}{ Drug } & Label claim(mg) & Amount found(mg) & Drug content (\%) \\
\hline Aliskiren & 150 & 149.56 & 99.71 \\
\hline Amlodipine & 5 & 5.05 & 100.97 \\
\hline Hydrochlorothiazide & 12.5 & 12.48 & 99.86 \\
\hline
\end{tabular}

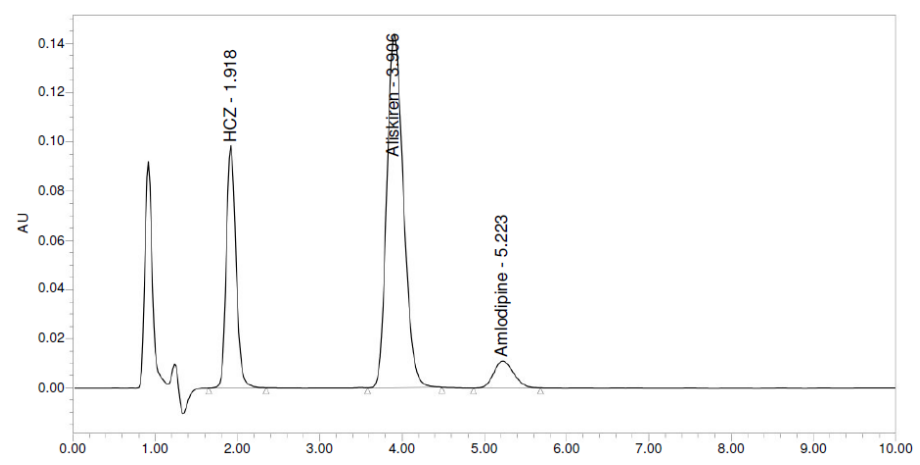

Fig. 4 Standard chromatogram of Aliskiren hemifumarate, Hydrochlorothiazide and Amlodipine

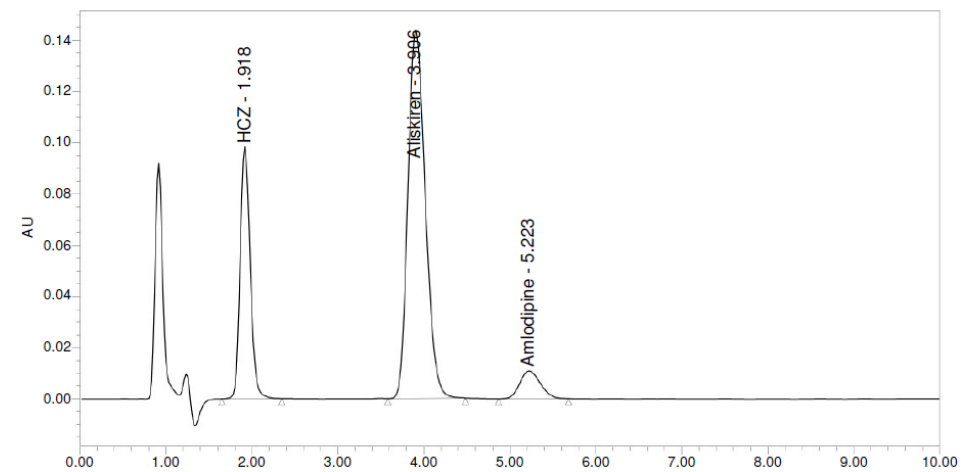

Fig. 5 Sample chromatogram of Aliskiren hemifumarate, Hydrochlorothiazide and Amlodipine

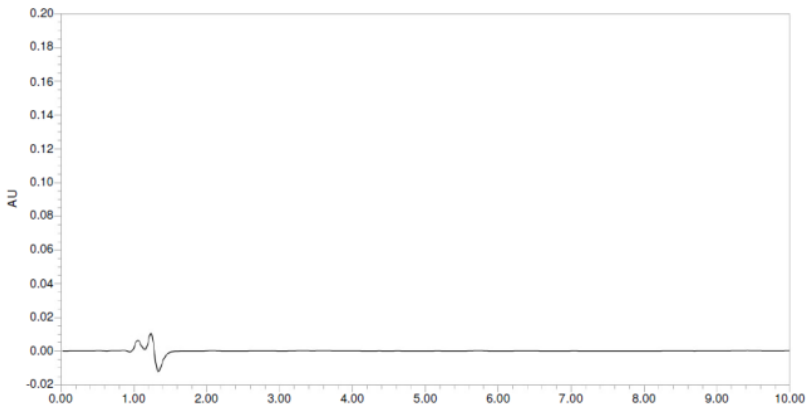

Fig. 6 Chromatogram of Blank

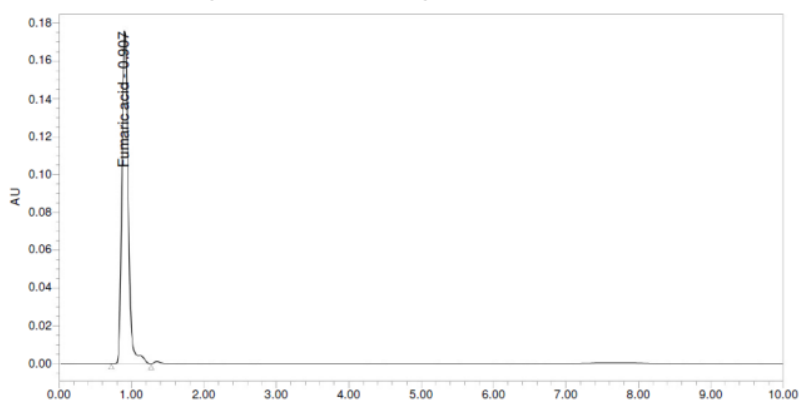

Fig. 7 Chromatogram of Fumaric acid 


\section{Method Validation}

After the method conditions were established as described above, method was validated as per ICH guidelines. The accuracy, precision, Linearity, limit of detection (LOD) and quantification (LOQ) were determined. These values are summarized in Table 10.

\subsection{Accuracy}

Accuracy was determined in terms of percentage recovery. Sample solution spiked with the analytes at three different concentration levels $37.5-225.00 \mu \mathrm{g} / \mathrm{ml}$ of Aliskiren hemifumarate, 3.125-18.75 $\mu \mathrm{g} / \mathrm{ml}$ of Hydrochlorothiazide and $1.25-7.50 \mu \mathrm{g} / \mathrm{ml}$ of Amlodipine. Another set of standard mixtures at the same concentration levels were also prepared with the diluents. Sample and standard solutions were injected into the HPLC system. Percentage recoveries of Aliskiren hemifumarate, Hydrochlorothiazide and Amlodipine were calculated. The values are summarized in Table 2.

Table 2: Recovery Studies

\begin{tabular}{|c|c|c|c|c|c|}
\hline \multirow{2}{*}{ Drug } & Concentration & $\begin{array}{c}\text { Peak area } \\
\text { (avg) }\end{array}$ & $\begin{array}{c}\text { Amount of } \\
\text { drug added }\end{array}$ & $\begin{array}{c}\text { Amount of drug } \\
\text { found }\end{array}$ & \% Recovery \\
\hline \multirow{3}{*}{ Aliskiren } & $80 \%$ & 1611146 & 120.10 & 120.16 & 100.05 \\
\cline { 2 - 6 } & $100 \%$ & 2025916 & 150.15 & 151.09 & 100.60 \\
\cline { 2 - 6 } & $120 \%$ & 2425139 & 180.90 & 180.86 & 99.98 \\
\hline \multirow{3}{*}{ Amlodipine } & $80 \%$ & 140674 & 6.05 & 6.06 & 99.67 \\
\cline { 2 - 6 } & $100 \%$ & 178060 & 5.08 & 5.13 & 100.93 \\
\cline { 2 - 6 } & $120 \%$ & 213714 & 4.00 & 3.99 & 100.11 \\
\cline { 2 - 6 } & $80 \%$ & 646293 & 10.01 & 10.09 & 100.84 \\
\cline { 2 - 6 } & $100 \%$ & 805837 & 12.50 & 12.59 & 100.69 \\
\hline
\end{tabular}

\subsection{Precision}

Method precision was determined both in terms of repeatability (injection and analysis) and intermediate precision (intra-day and inter-days reproducibility). In order to determine injection repeatability, samples spiked with Aliskiren hemifumarate, hydrochlorothiazide and amlodipine were injected 6 times into HPLC system and repeatability of the retention time and peak area were determined and expressed as mean and \%RSD calculated from the data obtained. The values are summarized in Table 3, 4, 5 and 6 .

Table 3: System Precision

\begin{tabular}{|l|c|c|c|c|c|c|}
\hline \multirow{2}{*}{ Name } & \multicolumn{2}{|c|}{ Hydrochlorothiazide } & \multicolumn{2}{c|}{ Aliskiren } & \multicolumn{2}{c|}{ Amlodipine } \\
\cline { 2 - 7 } & RT & Area & RT & Area & RT & Area \\
\hline System Precision-1 & 1.916 & 799421 & 3.896 & 2014646 & 5.207 & 177004 \\
\hline System Precision-2 & 1.912 & 794230 & 3.905 & 2002840 & 5.216 & 176608 \\
\hline System Precision-3 & 1.919 & 796920 & 3.907 & 2006750 & 5.222 & 178796 \\
\hline System Precision-4 & 1.915 & 797648 & 3.904 & 2017752 & 5.223 & 178685 \\
\hline System Precision-5 & 1.918 & 797648 & 3.906 & 2017752 & 5.223 & 178685 \\
\hline System Precision-6 & 1.914 & 794230 & 3.905 & 2002840 & 5.216 & 176608 \\
\hline Avg & 1.916 & 796683 & 3.904 & 2010430 & 5.218 & 177731 \\
\hline Std Dev & 0.003 & 2071.22 & 0.004 & 7124.00 & 0.006 & 1095.92 \\
\hline RSD & 0.135 & 0.260 & 0.102 & 0.354 & 0.120 & 0.617 \\
\hline
\end{tabular}

Table 4: Method Precision

\begin{tabular}{|l|c|c|c|c|c|c|}
\hline \multirow{2}{*}{ Name } & \multicolumn{2}{|c|}{ Hydrochlorothiazide } & \multicolumn{2}{c|}{ Aliskiren } & \multicolumn{2}{c|}{ Amlodipine } \\
\cline { 2 - 7 } & RT & Area & RT & Area & RT & Area \\
\hline Method Precision-1 & 1.912 & 796525 & 3.905 & 2006841 & 5.222 & 178796 \\
\hline Method Precision-2 & 1.916 & 797562 & 3.905 & 2017774 & 5.224 & 178692 \\
\hline Method Precision-3 & 1.912 & 794241 & 3.906 & 2010845 & 5.212 & 176625 \\
\hline Method Precision-4 & 1.915 & 797572 & 3.905 & 2016785 & 5.226 & 178794 \\
\hline Method Precision-5 & 1.916 & 797742 & 3.904 & 2017854 & 5.225 & 178881 \\
\hline Method Precision-6 & 1.918 & 797644 & 3.904 & 2017651 & 5.223 & 178781 \\
\hline Avg & 1.915 & 796881 & 3.905 & 2014625 & 5.222 & 178428 \\
\hline Std Dev & 0.002 & 1368.29 & 0.001 & 4669.99 & 0.005 & 885.40 \\
\hline RSD & 0.125 & 0.172 & 0.019 & 0.232 & 0.098 & 0.496 \\
\hline
\end{tabular}


Table 5: Interday Precision

\begin{tabular}{|c|c|c|c|c|c|c|}
\hline \multirow{2}{*}{ Name } & \multicolumn{2}{|c|}{ Hydrochlorothiazide } & \multicolumn{2}{|c|}{ Aliskiren } & \multicolumn{2}{|c|}{ Amlodipine } \\
\hline & RT & Area & RT & Area & RT & Area \\
\hline Injection -1 & 1.916 & 799421 & 3.896 & 2014646 & 5.207 & 177004 \\
\hline Injection -2 & 1.912 & 794230 & 3.905 & 2002840 & 5.216 & 176608 \\
\hline Injection -3 & 1.919 & 796920 & 3.907 & 2006750 & 5.222 & 178796 \\
\hline Injection -4 & 1.915 & 797648 & 3.904 & 2017752 & 5.223 & 178685 \\
\hline Injection -5 & 1.918 & 797648 & 3.906 & 2017752 & 5.223 & 178685 \\
\hline Injection -6 & 1.914 & 794230 & 3.905 & 2002840 & 5.216 & 176608 \\
\hline Avg & 1.916 & 796683 & 3.904 & 2010430 & 5.218 & 177731 \\
\hline Std Dev & 0.003 & 2071.22 & 0.004 & 7124.00 & 0.006 & 1095.92 \\
\hline RSD & 0.135 & 0.260 & 0.102 & 0.354 & 0.120 & 0.617 \\
\hline
\end{tabular}

Table 6: Intraday Precision

\begin{tabular}{|l|c|c|c|c|c|c|}
\hline \multirow{2}{*}{ Name } & \multicolumn{2}{|c|}{ Hydrochlorothiazide } & \multicolumn{2}{c|}{ Aliskiren } & \multicolumn{2}{c|}{ Amlodipine } \\
\cline { 2 - 7 } & RT & Area & RT & Area & RT & Area \\
\hline Injection -1 & 1.912 & 794465 & 3.901 & 2024665 & 5.211 & 178012 \\
\hline Injection -2 & 1.912 & 796241 & 3.908 & 2012651 & 5.221 & 176712 \\
\hline Injection -3 & 1.915 & 795845 & 3.904 & 2026541 & 5.220 & 178825 \\
\hline Injection -4 & 1.918 & 796651 & 3.905 & 2018742 & 5.208 & 178672 \\
\hline Injection -5 & 1.913 & 798045 & 3.908 & 2018754 & 5.212 & 178665 \\
\hline Injection -6 & 1.915 & 798625 & 3.902 & 2012856 & 5.215 & 177654 \\
\hline Avg & 1.914 & 796645 & 3.905 & 2019035 & 5.215 & 178090 \\
\hline Std Dev & 0.002 & 1512.35 & 0.003 & 5781.65 & 0.005 & 812.97 \\
\hline RSD & 0.121 & 0.190 & 0.075 & 0.286 & 0.099 & 0.456 \\
\hline
\end{tabular}

\subsection{Linearity}

The linearity of the method was established by spiking a series of standard mixtures of Aliskiren hemifumarate $(37.5-225.00 \mu \mathrm{g} / \mathrm{ml})$, Hydrochlorothiazide $(3.125-18.75 \mu \mathrm{g} / \mathrm{ml})$ and Amlodipine $(1.25-7.50 \mu \mathrm{g} / \mathrm{ml})$ and the above solutions are injected onto the HPLC system. The standard Calibration curve for Aliskiren hemifumarate (Fig. 8), Amlodipine (Fig. 9) and Hydrochlorothiazide (Fig. 10) was constructed by plotting their response ratios (ratios of the peak area of the analytes) against their respective concentrations. Linear regression was applied and slopes (a), intercept (b), correlation coefficient (r) were determined. The values are summarized in Table 7.

Table 7: Linearity

\begin{tabular}{|c|c|c|c|c|c|c|}
\hline Sl. no. & $\begin{array}{c}\text { Aliskiren } \\
(\mu \mathrm{g} / \mathrm{ml})\end{array}$ & Area & $\begin{array}{c}\text { Amlodipine } \\
(\mu \mathrm{g} / \mathrm{ml})\end{array}$ & Area & HCT $(\mu \mathrm{g} / \mathrm{ml})$ & Area \\
\hline 1. & 37.50 & 527267 & 1.25 & 44451 & 3.125 & 212852 \\
\hline 2. & 75.00 & 1014161 & 2.50 & 89006 & 6.250 & 405379 \\
\hline 3. & 112.50 & 1512403 & 3.75 & 132692 & 9.375 & 602830 \\
\hline 4. & 150.00 & 2005341 & 5.00 & 175598 & 12.500 & 795096 \\
\hline 5. & 187.50 & 2507191 & 6.25 & 221176 & 15.625 & 986734 \\
\hline 6. & 225.00 & 3025116 & 7.50 & 263987 & 18.750 & 1184882 \\
\hline
\end{tabular}




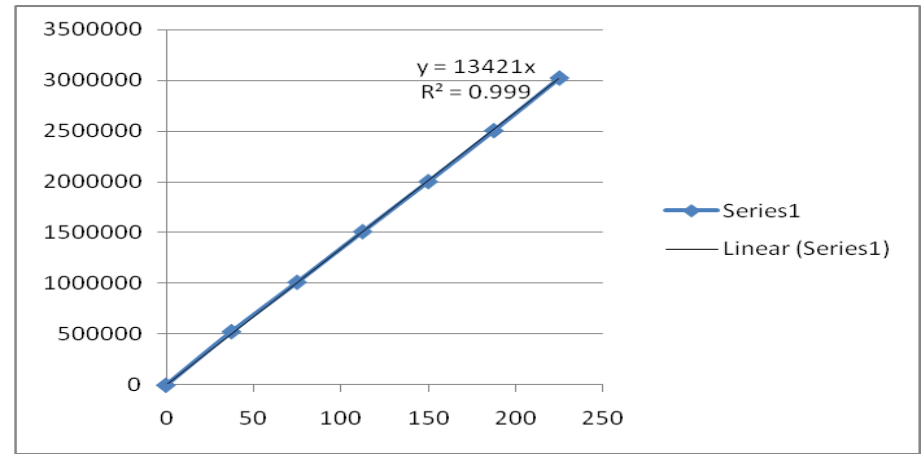

Figure 8: Standard calibration graph of Aliskiren

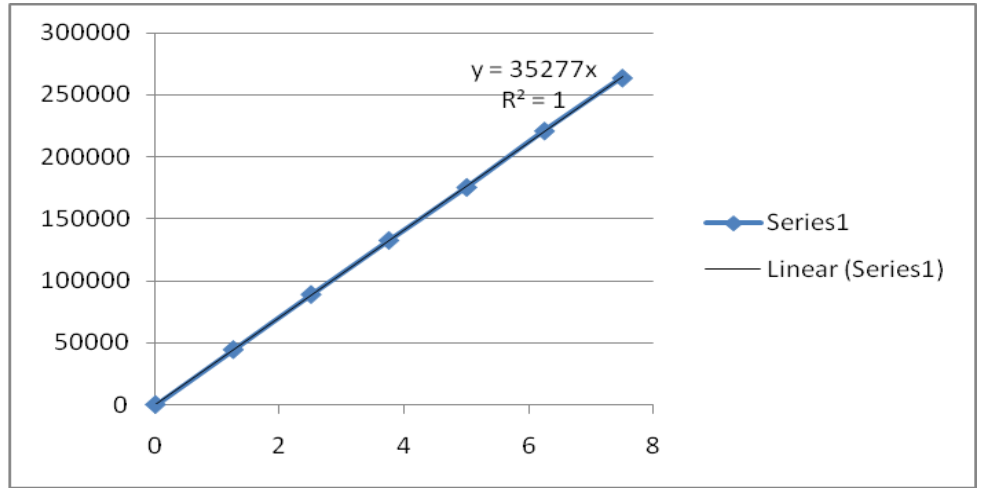

Figure 9: Standard calibration graph of Amlodipine

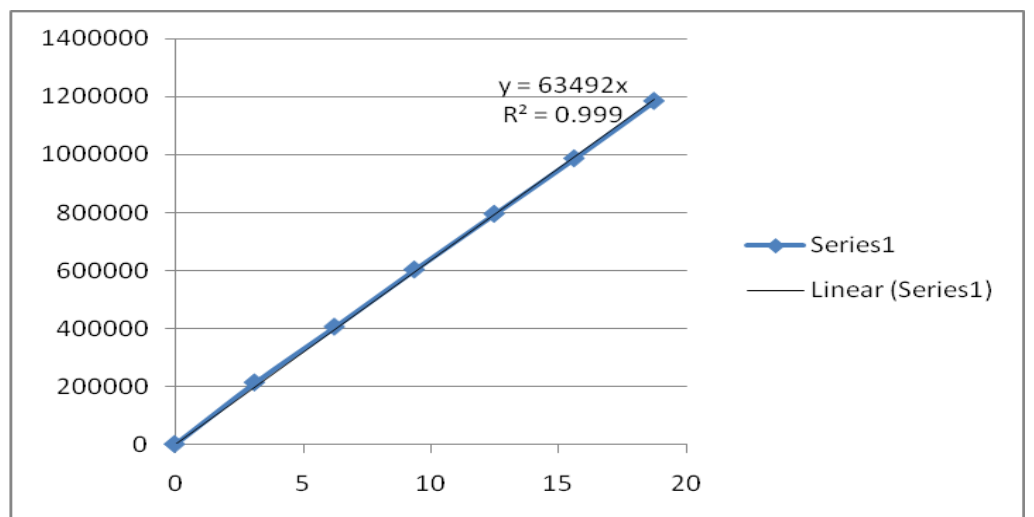

Figure 10: Standard calibration graph of Hydrochlorothiazide

\subsection{Limit of detection \& Limit of quantification}

Detection and quantification limits were determined through dilution method using $\mathrm{S} / \mathrm{N}$ approach by injecting a $20 \mu \mathrm{l}$ sample. LOD was considered as the minimum concentration with a signal to noise ratio of atleast three $\left(\mathrm{S}^{-} \mathrm{N}^{\sim} 3\right)$, while LOQ was taken as a minimum concentration with a signal to noise ratio of atleast ten $\left(\mathrm{S} / \mathrm{N}^{\sim} 10\right)$. The LOD for Aliskiren, Hydrochlorothiazide, Amlodipine standard solutions were found to be $2.886 \mathrm{ng} / \mathrm{ml}, 2.98 \mathrm{ng} / \mathrm{ml}$ and $2.99 \mathrm{ng} / \mathrm{ml}$ respectively. The LOQ for Aliskiren, Hydrochlorothiazide, Amlodipine standard solutions were found to be $9.79 \mathrm{ng} / \mathrm{ml}, 10.001 \mathrm{ng} / \mathrm{ml}$ and $9.98 \mathrm{ng} / \mathrm{ml}$ respectively (Table 10).

\subsection{Robustness}

As defined by ICH, The robustness of an analytical procedure describes to its capability to remain unaffected by small and deliberate variations in method parameters. Robustness was performed by small variation in the chromatographic conditions and found to be unaffected by small variations like flow rate $( \pm 10 \%)$, column oven temperature $\left( \pm 5^{\circ} \mathrm{c}\right)$ and wave length ( \pm 5 units). The values are summarized in Table 8 . 
Table 8: Robustness

\begin{tabular}{|c|c|c|c|}
\hline Drug & Flow rate & Column oven temperature & Wavelength \\
\hline \multirow{2}{*}{ Aliskiren } & $0.9 \mathrm{ml}$ & $25^{\circ} \mathrm{c}$ & $227 \mathrm{~nm}$ \\
& $1.1 \mathrm{ml}$ & $35^{\circ} \mathrm{c}$ & $237 \mathrm{~nm}$ \\
\hline \multirow{2}{*}{ Amlodipine } & $0.9 \mathrm{ml}$ & $25^{\circ} \mathrm{c}$ & $227 \mathrm{~nm}$ \\
& $1.1 \mathrm{ml}$ & $35^{\circ} \mathrm{c}$ & $237 \mathrm{~nm}$ \\
\hline \multirow{2}{*}{ Hydrochlorothiazide } & $0.9 \mathrm{ml}$ & $25^{\circ} \mathrm{c}$ & $227 \mathrm{~nm}$ \\
& $1.1 \mathrm{ml}$ & $35^{\circ} \mathrm{c}$ & $237 \mathrm{~nm}$ \\
\hline
\end{tabular}

\subsection{Ruggedness}

The method is rugged by different analyst, different time intervals and the method did not significantly affect the recoveries, peak area and retention time of all the above drugs indicating that the proposed method is rugged. The values are summarized in Table 9.

Table 9: Ruggedness

\begin{tabular}{|l|c|c|c|c|c|c|}
\hline \multirow{2}{*}{ Name } & \multicolumn{2}{|c|}{ Hydrochlorothiazide } & \multicolumn{2}{c|}{ Aliskiren } & \multicolumn{2}{c|}{ Amlodipine } \\
\cline { 2 - 7 } & RT & Area & RT & Area & RT & Area \\
\hline Injection -1 & 1.912 & 794465 & 3.901 & 2024665 & 5.211 & 178012 \\
\hline Injection -2 & 1.912 & 796241 & 3.908 & 2012651 & 5.221 & 176712 \\
\hline Injection -3 & 1.915 & 795845 & 3.904 & 2026541 & 5.220 & 178825 \\
\hline Injection -4 & 1.918 & 796651 & 3.905 & 2018742 & 5.208 & 178672 \\
\hline Injection -5 & 1.913 & 798045 & 3.908 & 2018754 & 5.212 & 178665 \\
\hline Injection -6 & 1.915 & 798625 & 3.902 & 2012856 & 5.215 & 177654 \\
\hline Avg & 1.914 & 796645 & 3.905 & 2019035 & 5.215 & 178090 \\
\hline Std Dev & 0.002 & 1512.35 & 0.003 & 5781.65 & 0.005 & 812.97 \\
\hline RSD & 0.121 & 0.190 & 0.075 & 0.286 & 0.099 & 0.456 \\
\hline
\end{tabular}

Table 10: Validation Parameters of the Method

\begin{tabular}{|l|c|c|c|}
\hline \multicolumn{1}{|c|}{ Method Parameters } & Aliskiren & Hmlodpine & Hydrochlorothiazide \\
\hline Linearity range $(\mu \mathrm{g} / \mathrm{ml})$ & $37.5-225.00$ & $1.25-7.50$ & $3.125-18.75$ \\
\hline Correlation coefficient & 0.999 & 1 & 0.999 \\
\hline LOD $(\mathrm{ng} / \mathrm{ml})$ & 2.88 & 9.99 & 2.98 \\
\hline LOQ $(\mathrm{ng} / \mathrm{ml})$ & 9.79 & 5.22 & 10.001 \\
\hline Retention time & 3.90 & 3454 & 3274 \\
\hline Theoretical plates & 3574 & 1.14 & 1.12 \\
\hline Tailing factor & 1.16 & 3.21 & 5.20 \\
\hline Resolution & 1.68 & 0.456 & 0.190 \\
\hline Precision $(\%$ RSD) & 0.286 & 0.099 & 0.121 \\
\hline Intra-day $(\mathrm{n}=3)$ & 0.075 & 0.120 & \\
\hline Inter-day $(\mathrm{n}=3)$ & 0.102 & 100.23 & \\
\hline$\%$ Recovery $(\mathrm{n}=6)$ & 100.21 & & \\
\hline
\end{tabular}

\section{Forced Degradation Studies (Stress Testing)}

Forced degradation studies were carried out for all the three drugs. The bulk drugs were subjected to alkaline studies by adding $1.0 \mathrm{ml}$ of $0.1 \mathrm{M} \mathrm{NaOH}$ for $4 \mathrm{hrs}$, $8 \mathrm{hrs}$ and $12 \mathrm{hrs}$ neutralized with $1.0 \mathrm{ml}$ of $0.1 \mathrm{M} \mathrm{HCl}$ acid. Similarly, the acidic studies were performed by adding $1.0 \mathrm{ml}$ of $0.1 \mathrm{M} \mathrm{HCl}$ for $4 \mathrm{hrs}$, $8 \mathrm{hrs}$ and $12 \mathrm{hrs}$ and neutralized with $1 \mathrm{ml}$ of $0.1 \mathrm{M} \mathrm{NaOH}$. Oxidation studies were performed on bulk drug by adding $1.0 \mathrm{ml}$ of $3 \%$ $\mathrm{H}_{2} \mathrm{O}_{2}$, thermal studies were performed by keeping the drug at $100^{\circ} \mathrm{C}$ and $\mathrm{UV}$ studies were performed with UVLamp for $4 \mathrm{hrs}, 8 \mathrm{hrs}$ and $12 \mathrm{hrs}$ respectively (Figure $11-15$ ). All samples were taken in different $10 \mathrm{ml}$ volumetric flask and dissolved in mobile phase. Final assay drug concentration was made up with mobile phase and injected in the chromatographic system. For all the stability study, the formation of degradable product was confirmed by comparing to chromatogram of the solution kept under normal conditions. All stressed samples were analyzed by developed HPLC method. The degradation data for Aliskiren hemifumarate, Amlodipine besylate and Hydrochlorothiazide was shown in Table 11, 12 \& 13 respectively. 


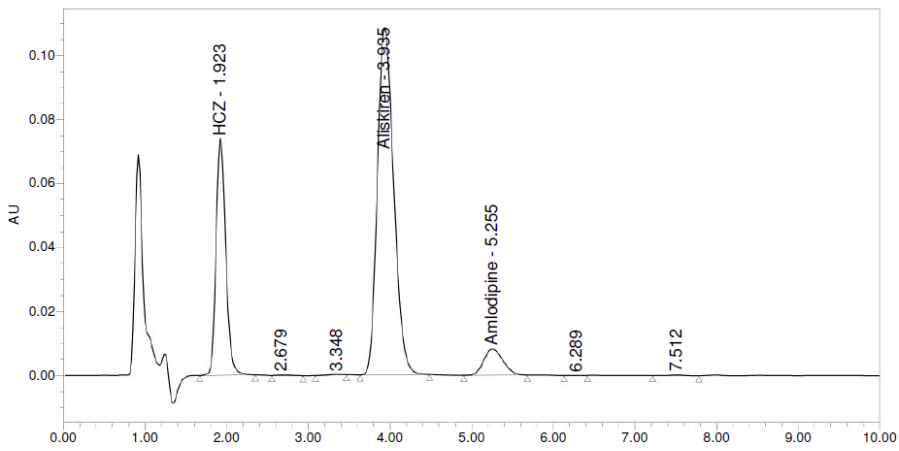

Figure 11: Typical chromatogram of Acid hydrolysis

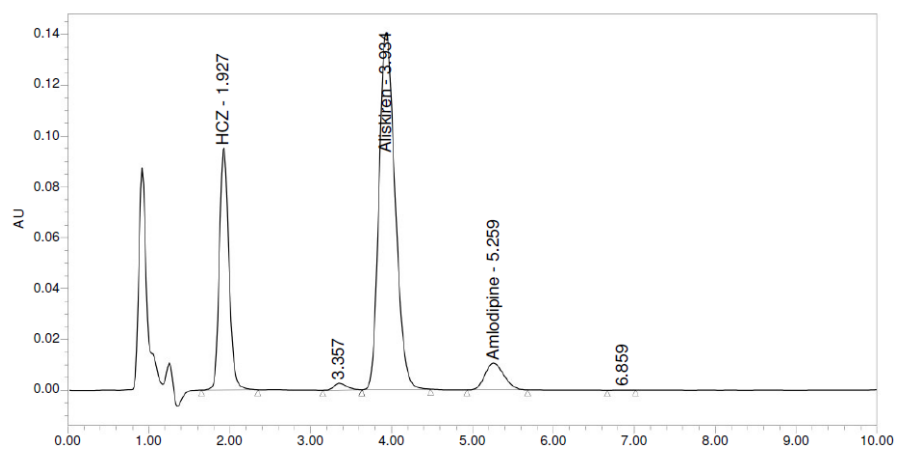

Figure 12: Typical chromatogram of Alkaline hydrolysis

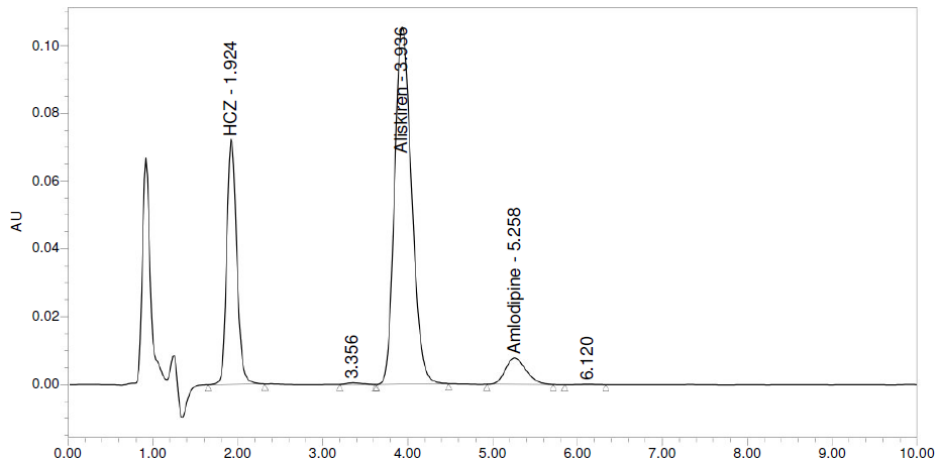

Figure 13: Typical chromatogram of Oxidation

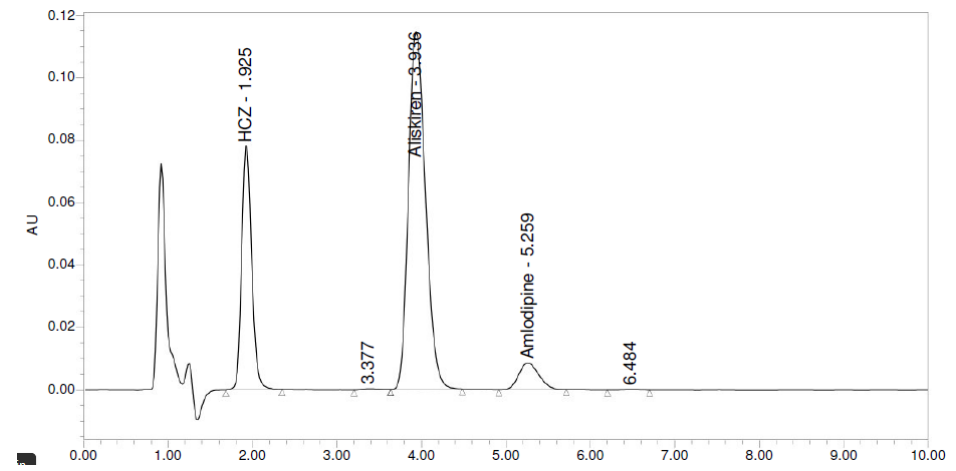

Figure 14: Typical chromatogram of UV 


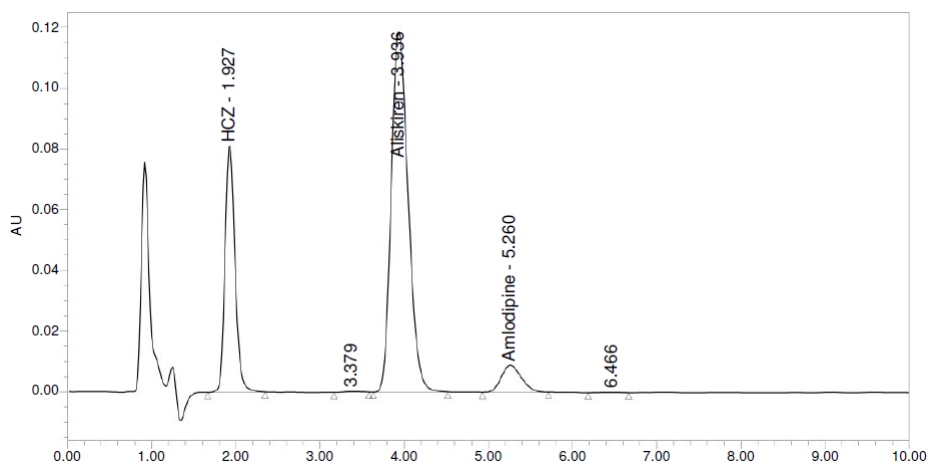

Figure 15: Typical Chromatogram of Thermal Degradation

Table 11: Degradation data for Aliskiren

\begin{tabular}{|l|c|c|}
\hline \multicolumn{1}{|c|}{ Stress condition } & Degradation time & Degradation (\%) \\
\hline \multirow{2}{*}{$\begin{array}{l}\text { Acidic } \\
(0.1 \mathrm{Hcl})\end{array}$} & $4 \mathrm{hrs}$ & 97.12 \\
\cline { 2 - 3 } & $8 \mathrm{hrs}$ & 93.76 \\
\cline { 2 - 3 } $\begin{array}{l}\text { Alkaline } \\
(0.1 \mathrm{NaOH})\end{array}$ & $12 \mathrm{hrs}$ & 90.17 \\
\hline \multirow{2}{*}{$\begin{array}{l}\text { Oxidation } \\
\left(\mathrm{H}_{2} \mathrm{O}_{2}\right)\end{array}$} & $4 \mathrm{hrs}$ & 98.09 \\
\cline { 2 - 3 } & $8 \mathrm{hrs}$ & 97.83 \\
\hline \multirow{2}{*}{$\mathrm{UV}$} & $12 \mathrm{hrs}$ & 95.06 \\
\cline { 2 - 3 } & $4 \mathrm{hrs}$ & 95.36 \\
\cline { 2 - 3 } & $8 \mathrm{hrs}$ & 89.14 \\
\hline \multirow{3}{*}{ Thermal } & $12 \mathrm{hrs}$ & 83.09 \\
\cline { 2 - 3 } & $4 \mathrm{hrs}$ & 96.89 \\
\cline { 2 - 3 } & $8 \mathrm{hrs}$ & 92.37 \\
\cline { 2 - 3 } & $12 \mathrm{hrs}$ & 88.99 \\
\cline { 2 - 3 } & $4 \mathrm{hrs}$ & 96.17 \\
\cline { 2 - 3 } & $8 \mathrm{hrs}$ & 93.67 \\
\hline
\end{tabular}

Table 12: Degradation data for Amlodipine

\begin{tabular}{|l|c|c|}
\hline \multicolumn{1}{|c|}{ Stress condition } & Degradation time & Degradation (\%) \\
\hline \multirow{3}{*}{$\begin{array}{l}\text { Acidic } \\
(0.1 \mathrm{Hcl})\end{array}$} & $4 \mathrm{hrs}$ & 97.38 \\
\cline { 2 - 3 } & $8 \mathrm{hrs}$ & 95.18 \\
\cline { 2 - 3 } $\begin{array}{l}\text { Alkaline } \\
(0.1 \mathrm{NaOH})\end{array}$ & $12 \mathrm{hrs}$ & 92.77 \\
\cline { 2 - 3 } & $4 \mathrm{hrs}$ & 98.9 \\
\cline { 2 - 3 } $\begin{array}{l}\text { Oxidation } \\
\left(\mathrm{H}_{2} \mathrm{O}_{2}\right)\end{array}$ & $8 \mathrm{hrs}$ & 97.71 \\
\hline \multirow{3}{*}{ Thermal } & $12 \mathrm{hrs}$ & 95.17 \\
\cline { 2 - 3 } & $4 \mathrm{hrs}$ & 98.09 \\
\hline \multirow{2}{*}{$\mathrm{UV}$} & $8 \mathrm{hrs}$ & 94.53 \\
\cline { 2 - 3 } & $12 \mathrm{hrs}$ & 90.26 \\
\cline { 2 - 3 } & $4 \mathrm{hrs}$ & 97.22 \\
\cline { 2 - 3 } & $8 \mathrm{hrs}$ & 95.62 \\
\cline { 2 - 3 } & $12 \mathrm{hrs}$ & 93.41 \\
\cline { 2 - 3 } & $4 \mathrm{hrs}$ & 96.48 \\
\hline
\end{tabular}

Table 13: Degradation data for Hydrochlorothiazide

\begin{tabular}{|l|c|c|}
\hline Stress condition & Degradation time & Degradation (\%) \\
\hline \multirow{2}{*}{$\begin{array}{l}\text { Acidic } \\
(0.1 \mathrm{Hcl})\end{array}$} & $4 \mathrm{hrs}$ & 96.73 \\
\cline { 2 - 3 } & $8 \mathrm{hrs}$ & 92.59 \\
\cline { 2 - 3 } & $12 \mathrm{hrs}$ & 88.83 \\
\hline \multirow{3}{*}{$\begin{array}{l}\text { Alkaline } \\
(0.1 \mathrm{NaOH})\end{array}$} & $4 \mathrm{hrs}$ & 99.12 \\
\hline \multirow{2}{*}{$\begin{array}{l}\text { Oxidation } \\
\left(\mathrm{H}_{2} \mathrm{O}_{2}\right)\end{array}$} & $8 \mathrm{hrs}$ & 98.01 \\
\cline { 2 - 3 } & $12 \mathrm{hrs}$ & 96.00 \\
\hline \multirow{3}{*}{ Thermal } & $4 \mathrm{hrs}$ & 95.49 \\
\cline { 2 - 3 } & $8 \mathrm{hrs}$ & 91.23 \\
\hline \multirow{2}{*}{$\mathrm{UV}$} & $12 \mathrm{hrs}$ & 86.48 \\
\cline { 2 - 3 } & $4 \mathrm{hrs}$ & 96.84 \\
\cline { 2 - 3 } & $8 \mathrm{hrs}$ & 92.17 \\
\cline { 2 - 3 } & $12 \mathrm{hrs}$ & 87.56 \\
\cline { 2 - 3 } & $4 \mathrm{hrs}$ & 97.36 \\
\cline { 2 - 3 } & $8 \mathrm{hrs}$ & 94.91 \\
\hline
\end{tabular}




\section{Conclusion}

A novel, simple, rapid and cost effective RP-HPLC method was successfully developed for simultaneous determination of Aliskiren Hemifumarate, amlodipine besylate and hydrochlorothiazide. The proposed method was optimized and validated for the various experimental parameters. Influence of $\mathrm{pH}$ of the mobile phase, column oven temperature and various particulate columns on the analysis of Aliskiren Hemifumarate, amlodipine besylate and hydrochlorothiazide was evaluated. All the analytes were well resolved and separated in less than $10 \mathrm{~min}$. The developed method is a stability indicating method and can be conveniently used by quality control outfits to determine the contents of Aliskiren Hemifumarate, amlodipine besylate and hydrochlorothiazide simultaneously in routine and stability samples. This method could be used for the analysis of the drugs in pharmaceutical preparations and routine laboratory analysis with slight modification in the extraction procedure. Overall, the proposed method provides high throughput for simultaneous determination of Aliskiren Hemifumarate, amlodipine besylate and hydrochlorothiazide with excellent accuracy, precision, selectivity and reproducibility.

\section{Acknowledgement}

The authors express their sincere thanks to the Principal, JSS College of Pharmacy, Mysore and JSS University, Mysore for providing the facilities to carry out the research work.

\section{References}

[1] V.ulvi and h. keski-hynnil. First-derivative UV spectrophotometric and high performance liquid chromatographic analysis of some thiazide diuretics in the presence of their photodecomposition products. Journal of Pharmaceutical \& Biomedical Analysis, Vol.12, No. 7, pp. 917-922, 1994

[2] Feng Gao, Mengliang Zhang, Xiangyong Cui, Zhonghua Wang, Yantong Sun, Jingkai Gu. Simultaneous quantitation of hydrochlorothiazide and metoprolol in human plasma by liquid chromatography-tandem mass spectrometry. Journal of Pharmaceutical and Biomedical Analysis 52 (2010) 149-154

[3] Palak V.Chokshi*,Karan J.Trivedi, Nishit S.Patel Development And Validation Of RP-HPLC Method For Analysis Of Aliskiren Hemifumarate And Valsartan In their Combination Tablet Dosage Form International Journal of ChemTech Research Vol.4, No.4, pg 1623-1627

[4] Micheli Wrasse-Sangoi, Leonardo Trevisan Secretti. Development and validation of an UV spectrophotometric method for the determination of aliskiren in tablets. Isabel Fração Diefenbach e Clarice Madalena Bueno Rolim. Quim. Nova, Vol. 33, No. 6, 1330-1334, 2010.

[5] Richa Sah* and Saahil Arora. Development and validation of a HPLC analytical assay method for amlodipine besylate tablets: A Potent Ca+2 channel blocker. Journal of Advanced Pharmacy Education \& Research 2 (3) 93-100 (2012).

[6] Stability indicating RP-HPLC method for simultaneous determination of amlodipine and benazepril hydrochloride from their combination drug product. Journal of Pharmaceutical and Biomedical Analysis 39 (2005) 147-155.

[7] A. Zarghi, S.M. Foroutan, A. Shafaati, A. Khoddam. Validated HPLC method for determination of amlodipine in human plasma and its application to pharmacokinetic studies. Journal of Chromatography, II -Farmaco 60 (2005) 789-792.

[8] SS Chitlange, Kiran Bagri and DM Sakarkar. Stability indicating RP- HPLC method for simultaneous estimation of valsartan and amlodipine in capsule formulation. Asian J. Research Chem. 1(1): July-Sept. 2008

[9] Safeer K, Anbarasi B, N.Senthil Kumar. Analytical Method Development and Validation of Amlodipine and Hydrochlorothiazide in combined dosage form by RP-HPLC. International Journal of ChemTech ResearchVol.2, No.1, pp 21-25, Jan-Mar 2010.

[10] A. Mohammad, N. Rezanour, M. Ansari Dogaheh, F. Ghorbani Bidkorbeh, M. Hashem, R.B. Walker. A stability-indicating high performance liquid chromatographic (HPLC) assay for the simultaneous determination of atorvastatin and amlodipine in commercial tablets. Journal of Chromatography B, 846 (2007) 215-221.

[11] B. R. Kadam and S. B. Bari*quantitative analysis of valsartan and hydrochlorothiazide in tablets by high performance thin-layer chromatography with ultraviolet absorption densitometry. Journal of pharmaceutical \& biomedical analysis, Vol. 12, No. 7, pp. 917-922, 1994

[12] Abdel Fattah M. E1 Walily, Saeid F. Belal, Eman A. Heaba Ali E Kersh. Simultaneous determination of enalapril maleate and hydrochlorothiazide by first-derivative ultraviolet spectrophotometry and HPLC. Journal of Pharmaceutical and Biomedical Analysis 13 (1995) 851-856.

[13] Erden Banoglu a, Yalc,ýn, zkan b, Okan Atay. Dissolution tests of benazepril-HCL and hydrochlorothiazide in commercial tablets comparison of spectroscopic and high performance liquid chromatography methods.Il Farmaco 55 (2000) $477-483$.

[14] Eda Satana, Sadi Altýnay, Nilgun Gunden Goger, Sibel A. Ozkan, Zuhre Senturk, simultaneous determination of valsartan and hydrochlorothiazide in tablets by first-derivative ultraviolet spectrophotometry and LC. Journal of Pharmaceutical and Biomedical Analysis 25 (2001) 1009-1013.

[15] V.P. Rane, J.N. Sangshetti, and D.B. Shinde. Simultaneous High-Performance Liquid Chromatographic Determination of Telmisartan and Hydrochlorothiazide in Pharmaceutical Preparation. Journal of Chromatographic Science, Vol. 46, November/December 2008. 